\title{
Prevalence of Chronic Nonspecific Low Back Pain and Its Associated Factors among Middle-Aged and Elderly People: An Analysis Based on Data from a Musculoskeletal Examination in Japan
}

\author{
Yoichi Iizuka, Haku Iizuka, Tokue Mieda, Daisuke Tsunoda, Tsuyoshi Sasaki, \\ Tsuyoshi Tajika, Atsushi Yamamoto, Kenji Takagishi \\ Department of Orthopedic Surgery, Gunma University Graduate School of Medicine, Maebashi, Japan
}

\begin{abstract}
Study Design: A cross-sectional study.
Purpose: To clarify the prevalence of chronic nonspecific low back pain (CNSLBP) and its associated factors among middle-aged and elderly Japanese individuals using data from a musculoskeletal examination conducted in general Japanese populations.

Overview of Literature: Most studies evaluating low back pain-associated factors have been conducted in Western countries, but they have not always evaluated CNSLBP.

Methods: We obtained data on 213 subjects aged $>50$ years who responded to a survey regarding age, gender, body mass index, lifestyle-related diseases (diabetes mellitus, hypertension, and hyperlipidemia), glucocorticoid use, smoking and alcohol-drinking habits, labor intensity, and chronic low back pain (CLBP) and underwent screening for lumbar spinal stenosis, evaluation for quality of life (QOL), and evaluation for specific spinal pathology via thoracolumbar spine X-rays. We investigated the prevalence of CNSLBP and association between CNSLBP and measured variables.

Results: The prevalence of CNSLBP and chronic specific low back pain (CSLBP) was $15.4 \%$ and $9.3 \%$, respectively. Among the subjects with CLBP, $62.2 \%$ had CNSLBP. In age-adjusted logistic models, smoking habits ( $p=0.049$, odds ratio [OR]=2.594), low back pain $(p<0.001,0 R=0.974)$, lumbar function $(p=0.001,0 R=0.967)$, and social function $(p=0.023,0 R=0.976)$ in the Japanese Orthopaedic Association Back Pain Evaluation Questionnaire (JOABPE0) were significantly associated with CNSLBP, whereas EQ-5D utility score $(p=0.024, \mathrm{OR}=0.068)$, low back pain $(p=0.007, \mathrm{OR}=0.981)$, lumbar function $(p=0.001, \mathrm{OR}=0.963)$, walking ability $(p=0.001,0 \mathrm{R}=0.968)$, and social function ( $p=0.002,0 R=0.966)$ in JOABPEQ were significantly associated with CSLBP.

Conclusions: CNSLBP among middle-aged and elderly individuals was associated with smoking habits and decreased QOL; however, CSLBP was considered to be more multilaterally associated decreased QOL.
\end{abstract}

Keywords: Prevalence; Low back pain; Life-style habits; Quality of life

Received Jan 31, 2017; Revised Mar 23, 2017; Accepted Mar 31, 2017

Corresponding author: Yoichi lizuka

Department of Orthopedic Surgery, Gunma University Graduate School of Medicine, 3-39-22 Showa, Maebashi, Gunma 371-8511, Japan

Tel: +81-27-220-8269, Fax: +81-27-220-8275, E-mail: yoiizuka@gunma-u.ac.jp 


\section{Introduction}

Low back pain is a highly prevalent complaint $[1,2]$ and is reportedly associated with decreased activities of daily living (ADLs) and quality of life (QOL) [3-5]. In addition, a specific diagnosis of low back pain cannot be obtained in approximately $80 \%$ patients with low back pain [6], indicating that patients with low back pain are often diagnosed with nonspecific low back pain.

Nonspecific low back pain is defined in the European guidelines for the management of chronic nonspecific low back pain (CNSLBP) as low back pain that is not attributable to a recognizable, specific pathology (e.g., infection, tumor, osteoporosis, fracture, structural deformity, and inflammatory diseases, such as ankylosing spondylitis, radicular syndrome, and cauda equina syndrome) [7]. Nonspecific low back pain is defined in the clinical practice guideline for the management of low back pain in Japan as low back pain without a plausible cause, similar to that in the European guidelines [8]. However, the definition of nonspecific low back pain in previous studies has not been uniform, and the exclusion of specific pathologies for low back pain has not been adequate in many previous studies on nonspecific low back pain.

To date, several factors including, physical workload [9], psychological factors [10-13], and cigarette smoking [1416], have been associated with low back pain. However, most studies on low back pain-associated factors have been conducted in Western countries, but they have not always evaluated CNSLBP.

The current study aimed to investigate the prevalence of CNSLBP and its associated factors after the exclusion of specific spinal pathologies using imaging modalities and screening tools for radicular syndrome and cauda equina syndrome in general middle-aged and elderly Japanese populations.

\section{Materials and Methods}

A medical examination for health-related diseases is conducted annually in Katashina Village (with a population of 4,465 in 2014) in Gunma Prefecture, Japan, and 1,037 people had participated in 2013. Among these participants, a musculoskeletal examination was additionally conducted in 581 participants, 213 (aged $\geq 50$ years) of whom were recruited for the present study ( 75 males and 138 females, average age: 66.7 years) based on the follow- ing inclusion criteria: (1) individuals whose basic information (age, gender, body mass index [BMI], presence of lifestyle-related diseases [diabetes mellitus, hypertension, and hyperlipidemia], glucocorticoid use, current smoking habits, current alcohol-drinking habits [ $\geq 3$ units/day], and labor intensity) was recorded, (2) individuals who answered a questionnaire on chronic low back pain (CLBP) that persisted for the previous 3 months, (3) individuals who underwent screening for lumbar spinal stenosis (LSS) based on the modified clinical diagnosis support tool for LSS (LSS-DST) [17,18], (4) individuals whose QOL was evaluated, (5) individuals who underwent an X-ray examination of the thoracolumbar spine, and (6) individuals who were informed about the current study and consented to participate.

If the subjects had a total score of $\geq 7$ in the screening for LSS using LSS-DST, they were diagnosed with LSS. QOL was evaluated using the Japanese version of the EuroQol-5 dimensions (EQ-5D) utility score, EuroQolvisual analog scale $[19,20]$, and the Japanese Orthopaedic Association Back Pain Evaluation Questionnaire (JOABPEQ) [21]. The JOABPEQ comprises five sections (25 items) related to low back pain, lumbar function, walking ability, social functioning, and mental health, and each section has a score ranging from 0 to 100 , with higher scores indicating a better condition.

In the current study, we defined CNSLBP as CLBP continuing for $\geq 3$ months with all of the following conditions: (1) no coronal deformity based on the Scoliosis Research Society-Schwab adult spinal deformity classification [22] (specifically, a Cobb angle of $<30^{\circ}$, as measured using an anteroposterior X-ray of the thoracolumbar spine), (2) no tumorous lesions detected on X-ray, (3) no infectious lesions detected on X-ray, (4) no vertebral fracture detected on X-ray, and (5) no LSS diagnosed using the LSS-DST.

The current study was approved by the Institutional Review Board of Gunma University (IRB NO. 23-31). All subjects provided written informed consent before participating in this study.

\section{Statistical Analyses}

Univariate analyses were conducted using the MannWhitney U test, chi-squared test, and Fisher's exact probability test. Multivariate analyses were also conducted after adjusting for age, a possible confounder, using logistic regression models. The significance level was set at 
$p<0.05$. All statistical analyses were conducted using the IBM SPSS Statistics ver. 22 software package (IMB Japan, Tokyo, Japan).

\section{Results}

Of the 213 subjects, 33 (15.5\%) and 20 (9.4\%) were diagnosed with CNSLBP and chronic specific low back pain (CSLBP), respectively, whereas the remaining 160 an-

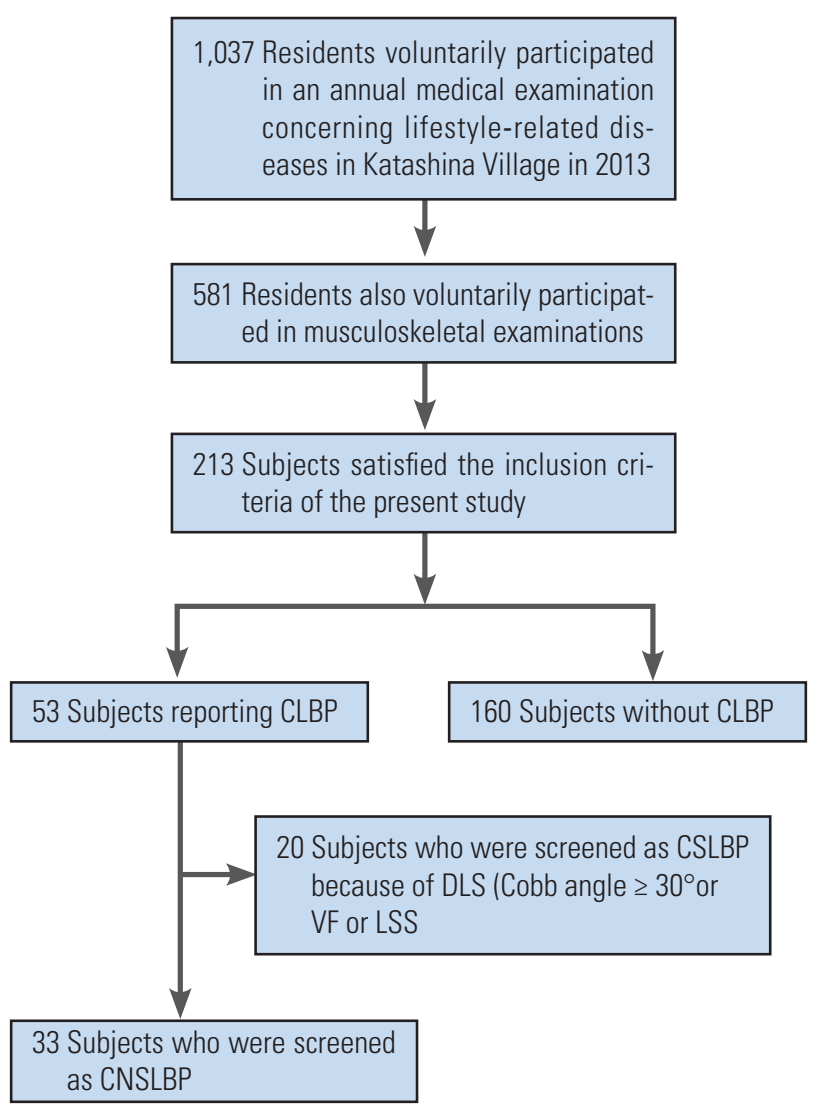

Fig. 1. Flowchart representing the screening results of chronic nonspecific low back pain and chronic specific low back pain. CLBP, chronic low back pain; CSLBP, chronic specific low back pain; DLS, degenerative lumbar scoliosis; VF, vertebral fracture; LSS, lumbar spinal stenosis; CNSLBP, chronic nonspecific low back pain. swered that they had no CLBP lasting for the previous $\geq 3$ months (Figs. 1, 2). Of the subjects with CLBP, $62.2 \%$ had CNSLBP. The prevalence of CNSLBP and CSLBP in each age group is shown in Table 1.

The univariate analysis comparing subjects with CNSLBP and those without CLBP revealed that age tended to be lower $(p<0.001)$ and the ratio of subjects with smoking habits $(p=0.011)$ was significantly higher among subjects with CNSLBP than among those without CLBP (Table 1). Furthermore, regarding QOL evaluation, the scores of EQ-5D $(p=0.016)$ and of two sections of the JOABPEQ, concerning low back pain $(p<0.001)$ and lumbar function $(p=0.001)$, were significantly lower in subjects with CNSLBP than in those without CLBP (Table $2)$. In age-adjusted logistic models, the presence of smoking habits $(p=0.049$, odds ratio $[\mathrm{OR}]=2.594)$ and scores of three sections of the JOABPEQ, concerning low back pain $(p<0.001, \mathrm{OR}=0.974)$, lumbar function $(p=0.001$, $\mathrm{OR}=0.967)$, and social function $(p=0.023, \mathrm{OR}=0.976)$, were significantly associated with CNSLBP (Table 3 ).

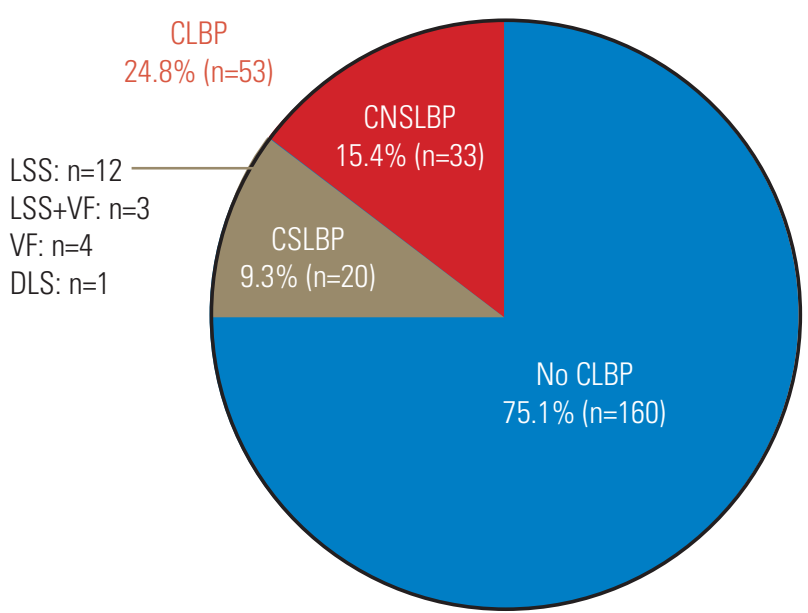

Fig. 2. Percentage of subjects with chronic low back pain, chronic nonspecific low back pain, and chronic specific low back pain. CLBP, chronic low back pain; CNSLBP, chronic nonspecific low back pain; CSLBP, chronic specific low back pain; LSS, lumbar spinal stenosis; VF, vertebral fracture, DLS, degenerative lumbar scoliosis.

Table 1. The prevalence of chronic nonspecific low back pain and chronic specific low back pain in each age group

\begin{tabular}{lccccc} 
Low back pain & 50 's $(n=62)$ & 60 's $(n=57)$ & 70 's $(n=73)$ & 80 's $(n=21)$ & Total $(n=213)$ \\
CNSLBP $(\%)$ & 27.4 & 22.8 & 4.1 & 0 & 15.5 \\
CSLBP $(\%)$ & 3.2 & 3.5 & 15.1 & 23.8 & 9.4 \\
No CLBP $(\%)$ & 69.4 & 73.7 & 80.8 & 76.2 & 75.1 \\
\hline
\end{tabular}

CNSLBP, chronic nonspecific low back pain; CSLBP, chronic specific low back pain. 
Table 2. The association between chronic nonspecific low back pain and the measured variables

\begin{tabular}{|c|c|c|c|}
\hline Variables & CNSLBP $(n=33)$ & No CLBP $(\mathrm{n}=160)$ & $p$-value \\
\hline Age (yr) & 60.5 & 67 & $<0.001^{\text {a) }}$ \\
\hline Sex (male/female) & $10 / 23$ & $60 / 100$ & $0.434^{\mathrm{bl}}$ \\
\hline Body mass index $\left(\mathrm{kg} / \mathrm{m}^{2}\right)$ & 24 & 23.2 & $0.422^{\text {a) }}$ \\
\hline Diabetes mellitus & $3(9.1)$ & $8(5.0)$ & $0.404^{c)}$ \\
\hline Hypertension & $8(24.2)$ & $58(36.3)$ & $0.229^{c)}$ \\
\hline Hyperlipidemia & $5(15.1)$ & $19(11.9)$ & $0.570^{c)}$ \\
\hline Glucocorticoid use & 0 & $1(0.6)$ & $>0.99^{c)}$ \\
\hline Smoking habit & $9(27.2)$ & $17(10.6)$ & $0.021^{\mathrm{cl}}$ \\
\hline Alcohol (3 or more units/day) & $12(36.3)$ & $47(29.3)$ & $0.428^{b)}$ \\
\hline Labor intensity (mild, 1; moderate, 2; severe, 3) & 2 & 1.9 & $0.965^{\text {a) }}$ \\
\hline \multicolumn{4}{|l|}{ HADS } \\
\hline HADS-A (0-21) & 11.1 & 10.7 & $0.349^{\mathrm{al}}$ \\
\hline HADS-D (0-21) & 11.5 & 11.5 & $0.751^{\text {a) }}$ \\
\hline \multicolumn{4}{|l|}{ EuroQol } \\
\hline EQ-5D utility score & 0.78 & 0.86 & $0.016^{a)}$ \\
\hline EQ-VAS score $(0-100)$ & 65.2 & 66.2 & $0.815^{\mathrm{al}}$ \\
\hline \multicolumn{4}{|l|}{ JOABPEQ scores } \\
\hline Low back pain & 60.1 & 83.3 & $<0.001^{\text {a) }}$ \\
\hline Lumbar function & 83.8 & 93.9 & $0.001^{a)}$ \\
\hline Walking ability & 92.5 & 93.6 & $0.399^{\mathrm{a})}$ \\
\hline Social function & 86.5 & 92.4 & $0.053^{\mathrm{al}}$ \\
\hline Mental health & 69.9 & 73.7 & $0.436^{a)}$ \\
\hline
\end{tabular}

Values are presented as mean value or number (\%).

CNSLBP, chronic nonspecific low back pain; CLBP, chronic low back pain; HADS-A, Hospital Anxiety and Depression Scale for anxiety; HADS-D, Hospital Anxiety and Depression Scale for depression; EQ-5D, EuroQol-5 dimensions; EQ-VAS, EuroQol-visual analogue scale; JOABPEQ, Japanese Orthopaedic Association Back Pain Evaluation Questionnaire.

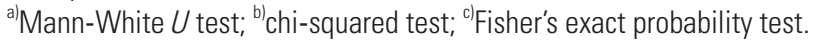

By contrast, the univariate analysis comparing subjects with CSLBP and those without CLBP revealed that age tended to be higher $(p=0.001)$ in subjects with CSLBP than in those without CLBP, and the scores of EQ-5D $(p=0.011)$ and four sections of the JOABPEQ, which concern low back pain $(p<0.001)$, lumbar function $(p<0.001)$, walking ability $(p<0.001)$, and social function $(p<0.001)$, were significantly lower in subjects with CSLBP than in those without CLBP (Table 4). In age-adjusted logistic models, the EQ-5D utility score $(p=0.024, \mathrm{OR}=0.068)$ and scores of four sections of the JOABPEQ, concerning low back pain $(p=0.007$, odd ratio $=0.981)$, lumbar function ( $p=0.001, \mathrm{OR}=0.963)$, walking ability $(p=0.001$, $\mathrm{OR}=0.968)$, and social function $(p=0.002, \mathrm{OR}=0.966)$, were significantly associated with CSLBP (Table 5 ).

\section{Discussion}

In the current study, we clarified the prevalence of CNSLBP and its associated factors in general middle-aged and elderly Japanese populations. To our knowledge, this is first study in which CNSLBP was diagnosed based on the exclusion of specific spinal pathologies using X-rays and LSS-DST.

Regarding the epidemiology of low back pain, Walker, in his systematic literature review, has reported that the point prevalence of low back pain in general ranges from $12 \%$ to $33 \%$, whereas its lifetime prevalence ranges from $11 \%$ to $84 \%$ [23]. By contrast, Bressler et al. [24], who systematically reviewed studies on the prevalence of low back pain in elderly individuals, have concluded that its prevalence in elderly population is not known with certainty. 
Table 3. The age-adjusted analysis for chronic nonspecific low back pain using logistic regression models

\begin{tabular}{|c|c|c|c|}
\hline Characteristic & Adjusted odds ratio & 95\% Confidence interval & $p$-value \\
\hline Sex (male/female) & 0.955 & $0.408-2.237$ & 0.916 \\
\hline Body mass index $\left(\mathrm{kg} / \mathrm{m}^{2}\right)$ & 1.05 & $0.944-1.167$ & 0.367 \\
\hline Diabetes mellitus & 2.606 & 0.589-11.534 & 0.207 \\
\hline Hypertension & 0.994 & $0.388-2.548$ & 0.99 \\
\hline Hyperlipidemia & 1.301 & $0.433-3.913$ & 0.639 \\
\hline Glucocorticoid use & 0 & 0 & - \\
\hline Smoking habit & 2.594 & $1.004-6.706$ & 0.049 \\
\hline Alcohol drinking habit & 1.311 & $0.580-2.965$ & 0.515 \\
\hline Labor intensity & 0.931 & $0.539-1.608$ & 0.797 \\
\hline \multicolumn{4}{|l|}{ HADS } \\
\hline HADS-A (0-21) & 1.126 & $0.908-1.397$ & 0.28 \\
\hline HADS-D (0-21) & 0.939 & $0.727-1.213$ & 0.631 \\
\hline \multicolumn{4}{|l|}{ Euro0ol } \\
\hline EQ-5D utility score & 0.181 & $0.028-1.195$ & 0.076 \\
\hline EQ-VAS score & 0.99 & $0.971-1.009$ & 0.285 \\
\hline \multicolumn{4}{|l|}{ JOABPEO scores } \\
\hline Low back pain & 0.974 & $0.961-0.986$ & $<0.001$ \\
\hline Lumbar function & 0.967 & $0.948-0.987$ & 0.001 \\
\hline Walking ability & 0.984 & $0.961-1.007$ & 0.179 \\
\hline Social function & 0.976 & $0.956-0.997$ & 0.023 \\
\hline Mental health & 0.985 & $0.963-1.008$ & 0.204 \\
\hline
\end{tabular}

HADS-A, Hospital Anxiety and Depression Scale for anxiety; HADS-D, Hospital Anxiety and Depression Scale for depression; EQ-5D, EuroQol-5 dimensions; EQ-VAS, EuroOol-visual analogue scale; JOABPEQ, Japanese Orthopaedic Association Back Pain Evaluation Questionnaire.

Furthermore, one study has reported that no scientific evidence on the prevalence of nonspecific low back pain has been published to date [7], and another has stated that a specific pathology cannot be identified in approximately $80 \%$ of patients with low back pain [6]. In the current study, the prevalence of CLBP and CNSLBP in a population of middle-aged and elderly Japanese individuals was $24.8 \%$ and $15.4 \%$, respectively. Furthermore, $62.2 \%$ of the subjects with CLBP had CNSLBP. The smaller percentage of subjects with CNSLBP among those with CLBP in the present study than that in previous reports may be because of differences in the race or mean age of the population. In addition, we also used a more exclusive definition of CNSLBP in the present study than that in previous reports, with the exclusion of specific pathologies [6].

Several factors associated with low back pain have been reported to date. Regarding lifestyle-related factors, physical workload [9] and cigarette smoking [14-16] have been reported to be associated with low back pain in general. However, no study has yet examined the factors associated with nonspecific low back pain by screening subjects with nonspecific low back pain using both X-ray and LSSDST. The univariate analysis in the current study revealed a significant association of younger age and smoking habits with CNSLBP, and the age-adjusted logistic regression analysis revealed a significant association of smoking habits with CNSLBP. Notably, gender, BMI, diabetes, hypertension, hyperlipidemia, glucocorticoid use, alcoholdrinking habits, and labor intensity were not significantly associated with CNSLBP in any analysis.

Several systematic review articles have identified an association between smoking habits and back pain [14-16]. However, a systematic review conducted by Wai et al. [25] has found that there is no evidence on whether smoking cessation relieves chronic low back pain. In his systematic review, Leboeuf-Yde [15] has concluded that smoking 
Table 4. The association between chronic specific low back pain and the measured variables

\begin{tabular}{|c|c|c|c|}
\hline Variables & $\operatorname{CSLBP}(\mathrm{n}=20)$ & No CLBP $(n=160)$ & $p$-value \\
\hline Age (yr) & 74.4 & 67 & $0.001^{\text {a) }}$ \\
\hline Sex (male/female) & $5 / 15$ & $60 / 100$ & $0.330^{b /}$ \\
\hline Body mass index $\left(\mathrm{kg} / \mathrm{m}^{2}\right)$ & 24.2 & 23.2 & $0.128^{\mathrm{a})}$ \\
\hline Diabetes mellitus & $1(50)$ & $8(5.0)$ & $>0.99^{b l}$ \\
\hline Hypertension & $7(35.0)$ & $58(36.3)$ & $>0.99^{b l}$ \\
\hline Hyperlipidemia & $3(15.0)$ & $19(11.9)$ & $0.716^{b)}$ \\
\hline Glucocorticoid use & $1(5.0)$ & $1(0.6)$ & $0.21^{\mathrm{b})}$ \\
\hline Smoking habit & 0 & $17(10.6)$ & $0.224^{\text {bl }}$ \\
\hline Alcohol (3 or more units/day) & $4(20.0)$ & $47(29.3)$ & $0.443^{b /}$ \\
\hline Labor intensity (mild, 1; moderate, 2; severe, 3) & 2 & 1.9 & $0.971^{\text {a) }}$ \\
\hline \multicolumn{4}{|l|}{ HADS } \\
\hline HADS-A (0-21) & 11.1 & 10.7 & $0.431^{\text {a) }}$ \\
\hline HADS-D (0-21) & 11.3 & 11.5 & $0.179^{\mathrm{al}}$ \\
\hline \multicolumn{4}{|l|}{ EuroQol } \\
\hline EQ-5D utility score & 0.76 & 0.86 & $0.011^{\text {a) }}$ \\
\hline EQ-VAS score (0-100) & 55.2 & 66.2 & $0.016^{a)}$ \\
\hline \multicolumn{4}{|l|}{ JOABPEQ scores } \\
\hline Low back pain & 61.3 & 83.3 & $<0.001^{\text {a) }}$ \\
\hline Lumbar function & 75.3 & 93.9 & $<0.001^{\text {a) }}$ \\
\hline Walking ability & 70 & 93.6 & $<0.001^{\text {a) }}$ \\
\hline Social function & 74.8 & 92.4 & $<0.001^{\text {a) }}$ \\
\hline Mental health & 66.8 & 73.7 & $0.051^{\text {a) }}$ \\
\hline
\end{tabular}

Values are presented as mean value or number (\%).

CSLBP, chronic specific low back pain; CLBP, chronic low back pain; HADS-A, Hospital Anxiety and Depression Scale for anxiety; HADS-D, Hospital Anxiety and Depression Scale for depression; EQ-5D, EuroOol-5 dimensions; EQ-VAS, EuroOol-visual analogue scale; JOABPE0, Japanese Orthopaedic Association Back Pain Evaluation Questionnaire.

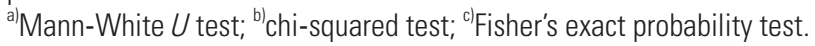

should be considered a weak indicator of low back pain, as evidence of a causal link between smoking and low back pain could not be found in a study using a large sample. In their systematic review, Goldberg et al. [14] have concluded that smoking might be associated with back pain, but additional longitudinal studies are needed to confirm the evidence. Shiri et al. [16], who assessed the association between smoking and low back pain in their meta-analysis, have concluded that the prevalence and incidence of low back pain are high in both current and former smokers, although the association is modest.

Although the precise pathophysiological role of smoking in the occurrence of low back pain has not been elucidated, several animal studies have been performed on this topic. Iwahashi et al. [26], who treated rabbits with nicotine levels equivalent to those encountered in heavy smokers, have concluded that nicotine treatment results in a delineation of the vascular buds in the vicinity of the vertebral end plate and a reduction in their number, leading to the degeneration of the intervertebral disc. Akmal et al. [27] have evaluated the effects of nicotine on nucleus pulposus cells using bovine intervertebral disc cells and have demonstrated that nicotine is associated with significant inhibition of cell proliferation and extracellular matrix synthesis in nucleus pulposus cells. Uei et al. [28] have compared the gene expression levels in the intervertebral discs between passively smoking rats and nonsmoking rats and have concluded that the changes in gene expression due to passive smoking precede the histologic changes in the intervertebral discs. Furthermore, Nemoto et al. [29] have investigated the histological changes in smoking-induced intervertebral disc degeneration after 
Table 5. The age-adjusted analysis for chronic specific low back pain using logistic regression models

\begin{tabular}{|c|c|c|c|}
\hline Characteristic & Adjusted odds ratio & $95 \%$ Confidence interval & $p$-value \\
\hline Sex (male/female) & 0.48 & $0.161-1.428$ & 0.187 \\
\hline Body mass index $\left(\mathrm{kg} / \mathrm{m}^{2}\right)$ & 1.133 & $0.967-1.327$ & 0.123 \\
\hline Diabetes mellitus & 0.697 & $0.080-6.116$ & 0.745 \\
\hline Hypertension & 0.489 & $0.169-1.418$ & 0.188 \\
\hline Hyperlipidemia & 1.402 & $0.355-5.532$ & 0.629 \\
\hline Glucocorticoid use & 7.262 & $0.374-140.878$ & 0.19 \\
\hline Smoking habit & 0 & 0 & 0.998 \\
\hline Alcohol 3 or more units/day & 0.743 & $0.228-2.426$ & 0.623 \\
\hline Labor intensity & 1.156 & $0.592-2.256$ & 0.671 \\
\hline \multicolumn{4}{|l|}{ HADS } \\
\hline HADS-A (0-21) & 1.169 & $0.891-1.534$ & 0.258 \\
\hline HADS-D (0-21) & 0.946 & $0.703-1.272$ & 0.713 \\
\hline \multicolumn{4}{|l|}{ EuroOol } \\
\hline EQ-5D utility score & 0.068 & $0.007-0.703$ & 0.024 \\
\hline EQ-VAS score & 0.977 & $0.953-1.002$ & 0.076 \\
\hline \multicolumn{4}{|l|}{ JOABPEQ scores } \\
\hline Low back pain & 0.981 & $0.967-0.995$ & 0.007 \\
\hline Lumbar function & 0.963 & $0.943-0.984$ & 0.001 \\
\hline Walking ability & 0.968 & $0.950-0.986$ & 0.001 \\
\hline Social function & 0.966 & $0.946-0.987$ & 0.002 \\
\hline Mental health & 0.975 & $0.945-1.005$ & 0.105 \\
\hline
\end{tabular}

HADS-A, Hospital Anxiety and Depression Scale for anxiety; HADS-D, Hospital Anxiety and Depression Scale for depression; EQ-5D, EuroQol-5 dimensions; EQ-VAS, EuroQol-visual analogue scale; JOABPEQ, Japanese Orthopaedic Association Back Pain Evaluation Questionnaire.

smoking cessation using rat models and have found that smoking-induced intervertebral degeneration can be repaired by smoking cessation. Based on these previous findings on the association of smoking with disc degeneration in animal models, we hypothesized that a considerable number of patients with CNSLBP can be diagnosed with discogenic pain if discography can be performed.

Nakamura et al. [5], who analyzed the effect of chronic pain, including low back pain, on ADLs, have demonstrated that basic ADLs, instrumental ADLs, and SF-36 QOL scores are associated with chronic pain. Hirano et al. [3] have also revealed that low back pain and knee pain are significant contributors to an individual's QOL. Furthermore, Muraki et al. [4] have reported that low back pain has a larger impact on QOL than knee pain and have indicated that low back pain with vertebral fractures is strongly associated with a decreased QOL. In the current study, CNSLBP as well as CSLBP were found to be significantly associated with several items concerning QOL/
ADLs. However, more of these items were associated with CSLBP than with CNSLBP.

The current study has several limitations. First, this study had a cross-sectional design with a relatively small population. Longitudinal studies in a larger number of subjects will be needed to confirm the observed associations between CNSLBP and the measured variables. Second, although we inquired about the presence or absence of CLBP, we did not define the area for CLBP. Therefore, the criteria for answering these questions might have differed among individuals. Third, magnetic resonance imaging was not used to detect the presence of any specific pathology, including tumorous lesions, infectious lesions, vertebral fractures, and LSS. Instead, we used X-rays and LSS-DST to detect these pathologies because magnetic resonance imaging can only be used to screen subjects suffering from symptoms associated with spinal stenosis, and a number of asymptomatic subjects have radiographic stenosis [30]. In addition, LSS-DST and modified 
LSS-DST reportedly have high sensitivity and specificity $[17,18]$. Fourth, we did not investigate the types of work performed by the subjects; however, we investigated the labor intensity in the subjects and identified no significant association. Katashina Village is a mountain village where agroforestry and tourism are the main industries. Thus, the fact that a relatively high percentage of the subjects were engaged in these industries might have influenced the prevalence of CLBP and its associated factors.

\section{Conclusions}

In our study using a general Japanese population, CNSLBP in middle-aged and elderly individuals was associated with smoking habits and decreased QOL; however, CSLBP was considered to be more multilaterally associated with decreased QOL.

\section{Conflict of Interest}

No potential conflict of interest relevant to this article was reported.

\section{References}

1. Chou R, Qaseem A, Snow V, et al. Diagnosis and treatment of low back pain: a joint clinical practice guideline from the American College of Physicians and the American Pain Society. Ann Intern Med 2007;147:478-91.

2. Yoshimura N, Akune T, Fujiwara S, et al. Prevalence of knee pain, lumbar pain and its coexistence in Japanese men and women: The Longitudinal Cohorts of Motor System Organ (LOCOMO) study. J Bone Miner Metab 2014;32:524-32.

3. Hirano K, Imagama S, Hasegawa Y, Ito Z, Muramoto A, Ishiguro N. Impact of low back pain, knee pain, and timed up-and-go test on quality of life in community-living people. J Orthop Sci 2014;19:164-71.

4. Muraki S, Akune T, Oka H, et al. Health-related quality of life in subjects with low back pain and knee pain in a population-based cohort study of Japanese men: the Research on Osteoarthritis Against Disability study. Spine (Phila Pa 1976) 2011;36:1312-9.

5. Nakamura M, Nishiwaki Y, Ushida T, Toyama Y. Prevalence and characteristics of chronic musculoskeletal pain in Japan. J Orthop Sci 2011;16:424-32.
6. Deyo RA. Diagnostic evaluation of LBP: reaching a specific diagnosis is often impossible. Arch Intern Med 2002;162:1444-7.

7. Airaksinen O, Brox JI, Cedraschi C, et al. Chapter 4. European guidelines for the management of chronic nonspecific low back pain. Eur Spine J 2006;15 Suppl 2:S192-300.

8. Japanese Orthopaedic Association. Clinical practice guideline for the management of low back pain. Tokyo: Nankodo Co.; 2012.

9. Hartvigsen J, Bakketeig LS, Leboeuf-Yde C, Engberg M, Lauritzen T. The association between physical workload and low back pain clouded by the "healthy worker" effect: population-based cross-sectional and 5 -year prospective questionnaire study. Spine (Phila Pa 1976) 2001;26:1788-92.

10. Grotle M, Vollestad NK, Brox JI. Clinical course and impact of fear-avoidance beliefs in low back pain: prospective cohort study of acute and chronic low back pain: II. Spine (Phila Pa 1976) 2006;31:1038-46.

11. Hartvigsen J, Frederiksen H, Christensen K. Physical and mental function and incident low back pain in seniors: a population-based two-year prospective study of 1387 Danish Twins aged 70 to 100 years. Spine (Phila Pa 1976) 2006;31:1628-32.

12. Kopec JA, Sayre EC, Esdaile JM. Predictors of back pain in a general population cohort. Spine (Phila $\mathrm{Pa}$ 1976) 2004;29:70-7.

13. Meyer T, Cooper J, Raspe H. Disabling low back pain and depressive symptoms in the community-dwelling elderly: a prospective study. Spine (Phila Pa 1976) 2007;32:2380-6.

14. Goldberg MS, Scott SC, Mayo NE. A review of the association between cigarette smoking and the development of nonspecific back pain and related outcomes. Spine (Phila Pa 1976) 2000;25:995-1014.

15. Leboeuf-Yde C. Smoking and low back pain: a systematic literature review of 41 journal articles reporting 47 epidemiologic studies. Spine (Phila Pa 1976) 1999;24:1463-70.

16. Shiri R, Karppinen J, Leino-Arjas P, Solovieva S, Viikari-Juntura E. The association between smoking and low back pain: a meta-analysis. Am J Med 2010;123:87.e7-35.

17. Konno S, Hayashino Y, Fukuhara S, et al. Development of a clinical diagnosis support tool to identify patients with lumbar spinal stenosis. Eur Spine J 
2007;16:1951-7.

18. Nikaido T, Sekiguchi M, Yonemoto K, et al. Can be $\mathrm{ABI}$ in the lumbar spinal stenosis diagnosis support tool substituted by palpation of posterior tibial artery? - a multicenter crosssectional study (DISTOPROJECT): GP86. Spine 2014;Supp 1:159.

19. EuroQol Group. EuroQol: a new facility for the measurement of health-related quality of life. Health Policy 1990;16:199-208.

20. Ikeda S, Ikegemi N; on behalf of the Japanese EuroQol Tariff Project. Health status in Japanese population: results from Japanese EuroQol Study. Iryou-toKagaku 1997;9:83-92

21. Fukui M, Chiba K, Kawakami M, et al. JOA back pain evaluation questionnaire: initial report. J Orthop Sci 2007;12:443-50.

22. Schwab F, Ungar B, Blondel B, et al. Scoliosis Research Society-Schwab adult spinal deformity classification: a validation study. Spine (Phila Pa 1976) 2012;37:1077-82.

23. Walker BF. The prevalence of low back pain: a systematic review of the literature from 1966 to 1998. J Spinal Disord 2000;13:205-17.

24. Bressler HB, Keyes WJ, Rochon PA, Badley E. The prevalence of low back pain in the elderly: a systematic review of the literature. Spine (Phila Pa 1976) 1999;24:1813-9.
25. Wai EK, Rodriguez S, Dagenais S, Hall H. Evidenceinformed management of chronic low back pain with physical activity, smoking cessation, and weight loss. Spine J 2008;8:195-202.

26. Iwahashi M, Matsuzaki H, Tokuhashi Y, Wakabayashi $\mathrm{K}$, Uematsu Y. Mechanism of intervertebral disc degeneration caused by nicotine in rabbits to explicate intervertebral disc disorders caused by smoking. Spine (Phila Pa 1976) 2002;27:1396-401.

27. Akmal M, Kesani A, Anand B, Singh A, Wiseman M, Goodship A. Effect of nicotine on spinal disc cells: a cellular mechanism for disc degeneration. Spine (Phila Pa 1976) 2004;29:568-75.

28. Uei H, Matsuzaki H, Oda H, Nakajima S, Tokuhashi Y, Esumi M. Gene expression changes in an early stage of intervertebral disc degeneration induced by passive cigarette smoking. Spine (Phila Pa 1976) 2006;31:510-4.

29. Nemoto Y, Matsuzaki H, Tokuhasi Y, et al. Histological changes in intervertebral discs after smoking and cessation: experimental study using a rat passive smoking model. J Orthop Sci 2006;11:191-7.

30. Ishimoto Y, Yoshimura N, Muraki S, et al. Associations between radiographic lumbar spinal stenosis and clinical symptoms in the general population: the Wakayama Spine Study. Osteoarthritis Cartilage 2013;21:783-8. 